\title{
ДИАГНОСТИЧЕСКИЙ КОМПЛЕКС ДЛЯ ЭФФЕКТИВНОГО КОНТРОЛЯ СОСТОЯНИЯ ОБМОТОК ВЫСОКОВОЛЬТНЫХ ТРАНСФОРМАТОРОВ
}

\author{
Лавринович Алексей Валериевич1, \\ lavrinovich86@yandex.ru
}

\author{
Мытников Алексей Владимирович², \\ mytnikov66@mail.ru \\ 1 Томский научно-исследовательский и проектный институт нести и газа, \\ Россия, 634027, г. Томск, пр. Мира, 72. \\ 2 Национальный исследовательский Томский политехнический университет, \\ Россия, 634050, г. Томск, пр. Ленина, 30.
}

\begin{abstract}
Актуальность исследования. Все стадии производства и переработки полезных ископаемых включают объемный технологический комплекс, доминирующая роль в котором принадлежит электроэнергетическим системам, обеспечивающим работу всех основных узлов и агрегатов. Существенный класс высоковольтного оборудования таких систем представляют распределительные силовые транссформаторы и шунтирующие реакторы. Выход из строя трансформаторного оборудования вызывает внеплановую остановку технологического цикла, аварийную ситуацию и финансовые потери. Этого можно избежать, обеспечив надежную работу электрооборудования путем проведения качественной диагностики, позволяющей оценить остаточный ресурс работы трансформатора или реактора. Стандартные технологии, такие как измерения сопротивления постоянному току, коэфффициента абсорбции и тангенса угла диэлектрических потерь, не позволяют обнаруживать дефрект обмотки на ранней стадии развития, а потому не могут считаться эффрективными. Практически во всех мировых энергосистемах перспективным и рекомендованным к применению считается комплекс FRA, представляющий собой техническую реализацию метода анализа частотных характеристик. Однако данная технология сталкивается с трудностями технического и организационного характера при внедрении в энергосистемах России. Приборная база и программное обеспечение не всегда соответствуют требованиям и нормам испытаний электрооборудования сетей РФ. Как результат имеет место высокий процент ошибочных заключений о результатах контроля состояния. Ситуацию осложняет тот фракт, что патент на использование технологии FRA принадлежит институтам США, Канады и ряда Европейских стран. Учитывая недружелюбную политическую ситуацию, ряд санкций и ограничений на экспорт техники и технологий, дальнейшее использование технологии FRA может оказаться проблематичным. Современная собственная технология, отвечающая требованиям сегодняшнего дня, в этой сфере в РФ отсутствует. Поэтому разработка надежного диагностического комплекса, учитывающего технические, климатические и инье особенности электроэнергетических систем РФ и позволяющего эффрективно проводить контроль состояния обмоток с учетом специфики отечественного трансформаторного оборудования, является актуальной задачей.

Цель. Исследовать эфффективность работы диагностического комплекса на основе зондирующих импульсов наносекундной длительности на реальном трансформаторном оборудовании. Провести сравнительный анализ результатов, контроля состояния обмоток трансформатора разработанного комплекса и комплекса FRA.

Методы. На реальном трансформаторе ТМ-160/10-У1 на высоковольтной фазе созданы дефекты обмотки, такие как короткозамкнутье витки и механическое смещение. При помощи разработанного диагностического комплекса зондирующий импульс наносекундной длительности подавался на обмотку одной из фаз. На соседней обмотке регистрировался импульсотклик. C помощью электронных осциллографов Tektronix muna TDS-1012 осуществлялась регистрация зондирующего импульса и импульса-отклика. Измерения проводились для тех же дефектных состояний обмотки с применением комплекса FRA. Результаты. На реальном трансформаторе экспериментально исследована эфффективность разработанного диагностического комплекса для контроля состоянии обмоток трансформаторов на основе наносекундных зондирующих импульсов. Показано, что эфффективность контроля состояния обмоток трансформатора при помощи разработанного комплекса оказывается не хуже, а в ряде случаев превосходит эфффективность комплекса FRA.
\end{abstract}

\section{Ключевые слова:}

Контроль состояния, импульсный метод, коммутационный импульс, дефект обмотки, эфрфективность диагностики.

\section{Введение}

Надежная работа энергетического оборудования является фундаментом, на котором основано эффективное функционирование всей технологической цепи добычи и переработки геологических ресурсов. Аварийный отказ одной единицы электрооборудования ведет к техническим проблемам и финансовым потерям. Применяемые методы контроля состояния трансформаторов не вполне соответствуют требованиям сегодняшнего дня и способны выявить развитый дефект, что не приемлемо на современном этапе развития индустрии [1-4]. Особенно это относится к выявлению дефектов обмоток трансформаторов [2-5]. Технология импульсного дефектографирования является наиболее эффективным методом и позволяет выявлять дефекты обмотки на ранних стадиях развития [6-8]. Данный метод успешно развивался как в стенах научной лаборатории [7-9], так и в практике применения в энергосистемах [9-11]. Не смотря на интенсивное развитие в 1970-1980 гг. в бывшем СССР, разработки в области указанной технологии были прекращены по ряду технических, организаци- 
онных и финансовых причин. В настоящее время практически во всех западных энергосистемах интенсивно развивается технология частотного анализа или FRA (frequency-response analysis), которая реализуется с помощью диагностических комплексов FRA [12-18]. Более того, данный метод контроля состояния обмоток трансформаторов признан как наиболее чувствительный и рекомендован к применению соответствующим комитетом СИГРЭ [19]. Метод частотного анализа (МЧА) внедряется в энергосистемах России, однако при его адаптации имеет место ряд проблем [20]. К тому же необходимо принимать в расчет факт, что технология FRA запатентована в лабораториях стран Северной Америки, приборная база производится в странах Евросоюза. Учитывая непростую политическую ситуацию и ряд постоянно расширяющихся ограничений, велика вероятность полного прекращения и невозможности использования технологии FRA в энергосистемах России.

Однако результаты, полученные в лаборатории техники высоких напряжений Инженерной школы энергетики ТПУ, подтверждают тот факт, что импульсный метод диагностики обмоток незаслуженно забыт. Он не исчерпал своего потенциала и не уступает по эффективности технологии FRA [21-24]. Поэтому реализация импульсного метода контроля состояния обмоток на основе зондирующего импульса наносекундной длительности в виде диагностического комплекса является важной задачей, решению которой посвящена настоящая статья.

\section{Постановка задачи исследования}

Разработать диагностический комплекс для высокоэффективного контроля состояния обмок высоковольтных трансформаторов на основе зондирующих импульсов наносекундной длительности. Исследовать эффективность работы разработанного комплекса на реальном трансформаторном оборудовании. Провести сравнительный анализ результатов, контроля состояния обмоток трансформатора разработанного комплекса и комплекса FRA, который принят в качестве основной технологии в ведущих энергосистемах Америки, Азии, Австралии и Евросоюза.

Теория и принцип методов низковольтных импульсов и метода частотного анализа

Метод низковольтных импульсов (НВИ) применяется в электроэнергетических системах СССР с начала 1970-х гг.

Методика измерений состоит в подаче прямоугольного зондирующего импульса напряжением 100-500 В на одну из обмоток или в нейтраль трансформатора от специального генератора. Одновременно происходит осциллографирование реакции обмоток на воздействие приложенного импульса - снимается осциллограмма напряжения на измерительных сопротивлениях, которые подключены к другим обмоткам. Основой метода является принцип последовательного импульсного дефектографирования. Вначале происходит снятие нормограмм с заведомо здорового трансформатора на заводе-изготовителе или перед вводом в эксплуатацию. В дальнейшем их сравнивают с дефектограммами - осциллограммами, полученными при последующих измерениях после ввода трансформатора в эксплуатацию. Состояние обмоток трансформатора определяют путем сравнения между собой нормограмм и дефектограмм по определенной методике. Отклонения дефектограммы от нормограммы свидетельствуют о наличии электрических повреждений или механических деформаций обмоток. Если нормограммы отсутствуют, то анализ состояния обмоток проводится путем сравнения осциллограмм разных фаз трансформатора. Эффективность метода НВИ для обнаружения остаточных деформаций обмоток силовых трансформаторов объясняется его большой чувствительностью к изменениям геометрических размеров. Даже небольшие механические перемещения в обмотках существенно изменяют емкость отдельных элементов (межвитковые, межкатушечные), а существенные деформации приводят к изменениям индуктивности деформированных элементов. Все это изменяет собственные частоты колебаний, что фиксируется на осциллограммах импульсных токов и напряжений [6-10].

Не смотря на высокую (по сравнению с другими методами) чувствительность метода НВИ и его длительное совершенствование (в период 1966-1988 гг.), его погрешность оставалась в ряде случаев неудовлетворительной для практического применения. Это объяснялось неточностью и субъективизмом при анализе результатов дефектографирования. Испытания трансформаторов на динамических стендах во Всесоюзном Электротехническом Институте подтвердили, что механические повреждения обмоток трансформаторов вследствие электродинамических воздействий могут сопровождаться значительно мень-

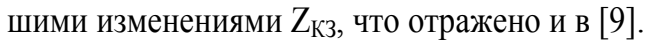

Преимущества метода НВИ: очень высокая чувствительность к большому количеству повреждений; возможность определять не только фазу, но и обмотку, в которой произошло повреждение.

К недостаткам метода НВИ следует отнести то, что высокая воспроизводимость результатов измерений возможна только при обеспечении полной идентичности измерений, интервал времени между которыми может составлять годы: схема и процедура измерений, используемые кабели и соединители, их взаимное расположение при испытаниях. Интерпретация результатов измерений требует высокой квалификации обслуживающего персонала [8-10]. Метод НВИ не позволяет количественно оценить возникшие остаточные деформации, требует отключения и расшиновки трансформатора, что не дает возможности применять системы мониторинга под рабочим напряжением, контролирующие механическое состояние обмоток при протекании сквозных токов КЗ [10].

Метод анализа частотных характеристик разработан в Канаде в 1978 г. в фирме «Онтарио - Гидро». МЧА (в англоязычной литературе - FRA от Frequency Response Analysis) основан на сравнении полученной в данный момент частотной характеристики обмотки с характеристикой, снятой прежде на том же или на 
однотипном трансформаторе. Процедура измерений по методу частотного анализа предполагает запись приложенного импульса и соответствующего ему отклика с использованием высокоточных аналогоцифровых преобразователей (АЦП). После чего результаты преобразуются в частотную область с помощью алгоритма быстрого преобразования Фурье. Рассчитанная таким образом передаточная функция зависит только от параметров испытуемого объекта и не зависит от приложенного сигнала и схемы измерений. Поэтому изменения в объекте могут быть отчетливо идентифицированы и отделены от проявлений внешних факторов, что существенно облегчает анализ результатов [16-19].

Параллельно развивалось другое направление данного метода - вместо импульсного воздействия на ввод обмотки подавалось синусоидальное напряжение, которое изменяется по частоте в широком диапазоне, а с других вводов снималось напряжение отклика. По результатам строилась амплитудно-частотная характеристика - реакция обмоток на воздействие. Оба способа получили достаточно широкое распространение.

Отклики обмоток анализируются по следующим проявлениям:

- различия между сигналами-откликами отдельных фаз трансформатора;

- различия между сигналами-откликами трансформаторов аналогичной конструкции;

- изменения амплитудно-частотных характеристик, полученных на одном трансформаторе, но в разное время.

Двухканальный аналого-цифровой преобразователь записывает по каналу № 1 сигнал, который подаётся на ввод обмотки, а по каналу № 2 - реакцию обмотки на воздействие. После этого рассчитывается передаточная функция. Передаточная функция представляет собой отношение спектров входного и выходного сигналов. Степень отличия передаточных функций, рассчитанных до и после воздействия на трансформатор электродинамических сил короткого замыкания или других механических воздействий, производится с помощью разных средств анализа.

Не смотря на безусловные достоинства метода FRA, процент ошибок остается довольно высоким. В ряде случаев при наличии дефекта был поставлен диагноз «здоров» и дефект не был распознан. В то же время случались ситуации, при которых трансформатор с исправными обмотками был забракован и выведен в ремонт.

\section{Исследование эффективности разработанного диагностического комплекса на реальном трансформаторном оборудовании}

Для установления степени чувствительности и достоверности контроля состояния и потенциальной производственной эффективности в условиях реальной энергосистемы были проведены исследования на реальном трансформаторе. Исследования состояли в обследовании исправного и дефектного трансформатора одинаковой марки, выпущенных на одном и том же предприятии, эксплуатируемых в практически одинаковых условиях, как разработанным методом наносекундных импульсов, так и методом анализа частотных характеристик.

Силовые трансформаторы марки ТМ-160/10-У1 находились в цехе по ремонту оборудования Ишимского ТПО, ОАО «Тюменьэнерго», г. Ишим, Тюменской области. Основные данные исследуемых трансформаторов приведены в табл. 1.

Таблица 1. Исходные данные объекта обследования

Table 1. Data of investigated object

\begin{tabular}{|c|c|}
\hline $\begin{array}{l}\text { Наименование } \\
\text { Name of option }\end{array}$ & $\begin{array}{c}\text { Данные объекта } \\
\text { Data of investigated object }\end{array}$ \\
\hline $\begin{array}{l}\text { Место расположения } \\
\text { трансформатора } \\
\text { Transformer location }\end{array}$ & $\begin{array}{c}\text { Цех по ремонту оборудования Ишимско- } \\
\text { го предприятия «Тюменьэнерго», } \\
\text { г. Ишим, Тюменской области } \\
\text { Ishim repair plant «Туumenenegro», } \\
\text { Ishim, Tyumen region }\end{array}$ \\
\hline $\begin{array}{l}\text { Наименование объекта } \\
\text { Call of object }\end{array}$ & $\begin{array}{c}\text { Tрансформатор } \\
\text { Transformer }\end{array}$ \\
\hline $\begin{array}{l}\text { Тип трансформатора } \\
\text { Type of transformer }\end{array}$ & $\begin{array}{l}\text { TM-160/10-У1 } \\
\text { TM-160/10-U1 }\end{array}$ \\
\hline $\begin{array}{l}\text { Дата выпуска, } \\
\text { завод изготовитель } \\
\text { Date of issue, } \\
\text { Production plant }\end{array}$ & $\begin{array}{c}1993 \text { г., } \\
\text { «Алтайский трансформатор», г. Барнаул } \\
\text { «Altay transformer», Barnaul }\end{array}$ \\
\hline $\begin{array}{l}\text { Дата ввода } \\
\text { в эксплуатацию } \\
\text { Date of work beginning }\end{array}$ & 1993 \\
\hline $\begin{array}{l}\text { Схема соединения } \\
\text { обмоток } \\
\text { Winding connection } \\
\text { scheme }\end{array}$ & $\mathrm{Y} / \mathrm{Y}_{0}$ \\
\hline $\begin{array}{l}\text { Номинальная } \\
\text { мощность } \\
\text { трансформатора } \\
\text { Transformer power } \\
\end{array}$ & 160 кВА (kVA) \\
\hline $\begin{array}{l}\text { Номинальное напря- } \\
\text { жение обмотки высо- } \\
\text { кого напряжения } \\
\text { Operating voltage of } \\
\text { high voltage winding }\end{array}$ & 10 кВ (kV) \\
\hline $\begin{array}{l}\text { Номинальное напря- } \\
\text { жение обмотки низко- } \\
\text { го напряжения } \\
\text { Operating voltage of } \\
\text { low voltage winding }\end{array}$ & 0,4 кB (kV) \\
\hline $\begin{array}{l}\text { Вид охлаждения } \\
\text { Cooling type }\end{array}$ & $\begin{array}{c}\text { Масляный } \\
\text { Oil type }\end{array}$ \\
\hline $\begin{array}{l}\text { Проблемные ситуации } \\
\text { во время эксплуатации } \\
\text { Problem situation during } \\
\text { work }\end{array}$ & $\begin{array}{l}\text { В результате грозы было повреждение } \\
\text { обмотки высокого напряжения, затем } \\
\text { трансформатор отремонтирован в ре- } \\
\text { монтном цехе Ишимского завода } \\
\text { As result of lightning, the high voltage } \\
\text { winding has been damaged. Than the trans- } \\
\text { former was repaired at the Ishim plant }\end{array}$ \\
\hline $\begin{array}{l}\text { Полная масса } \\
\text { Total mass }\end{array}$ & 800 кг (kg) \\
\hline $\begin{array}{l}\text { Macca масла } \\
\text { Oil mass }\end{array}$ & 225 кг (kg) \\
\hline
\end{tabular}

Внешний вид исследуемых трансформаторов, как исправного, так и с дефектным состоянием обмоток, приведен на рис. 1, 2, соответственно.

Основная цель исследования состояла в осуществлении контроля обмоток трансформаторов в исправном и дефектном состояниях двумя методами, а именно: методом наносекундных импульсов и FRA; сопоставлении и анализе полученных результатов. 


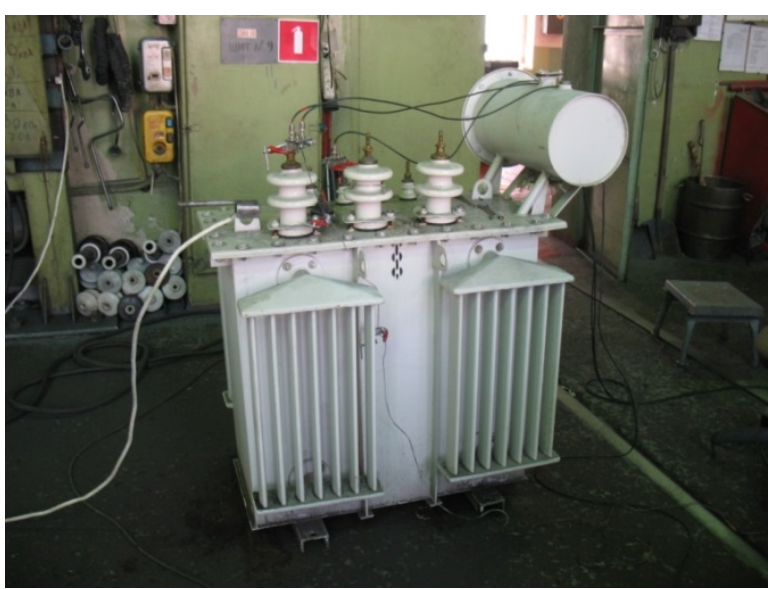

Pис. 1. Внешний вид обследуемого исправного трансформатора

Fig. 1. View of investigated transformer with normal condition

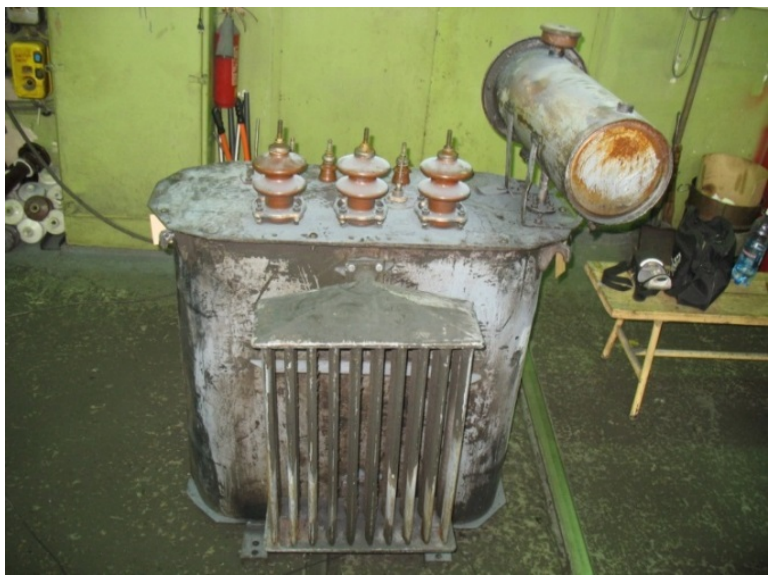

Pис. 2. Внешний вид обследуемого трансформатора с дефектным состоянием обмоток

Fig. 2. View of investigated transformer with failed windings

Схема контроля состояния обмоток трансформатора включающая генератор зондирующих импульсов наносекундного диапазона, осциллографы, контролирующие параметры зондирующего импульса и сигналы отклика, расположение фаз обмоток низкого напряжения $(\mathrm{HH})$ и высокого напряжения (BН), показана на рис. 3. В процессе измерений переключатель без возбуждения (ПБВ) находился в положении 2. Количество витков обмотки ВН - 1312, количество витков обмотки НН - 160 .

Процедура контроля состояния обмоток состояла в подаче на одну из обмоток зондирующего импульса $200 \mathrm{~B}, 520-25$ нс, и снятия сигнала отклика соответствующему переходному процессу с другой обмотки. В случае использования метода FRA применялся приборный комплекс FRAX-150, на экране которого происходило отображение амплитудно-частотных зависимостей, предварительно обработанных с помощью программно-аппаратных средств.

Основная цель экспериментов, результаты которых представлены ниже, выявить эффективность и провести сравнительный анализ разрабатываемого метода с методом FRA. Ниже приводится результат сравнения двух сигналов отклика при различных ситуациях в обмотках или изменений в объеме транс- форматора в целом. Сравнение результатов измерений выполнялось с использованием специальной программы, разработанной для указанных целей.

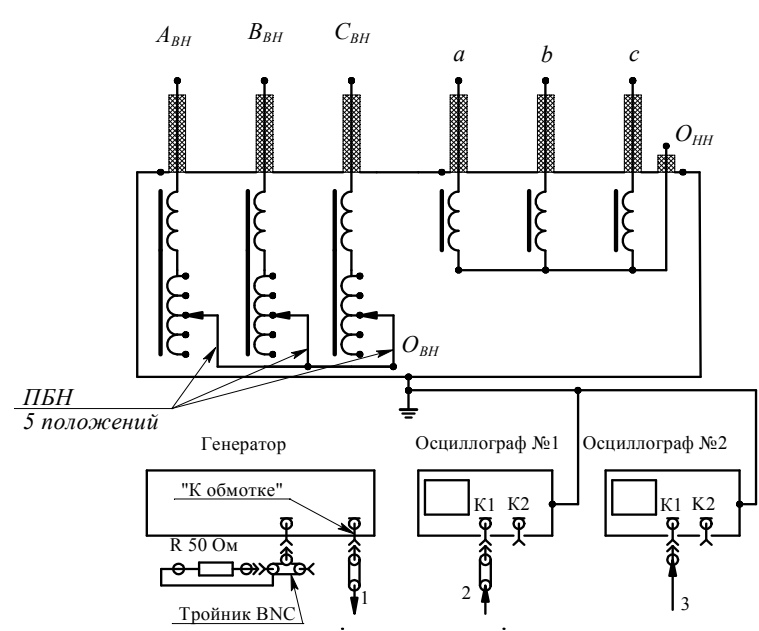

Pис. 3. Схема подключения приборов контроля при обследовании трансформатора

Fig. 3. Scheme of measuring devices connection at the winding control

Выбор критерия эффективности. Одним из основных критериев эффективности метода контроля состояния является его чувствительность. Чувствительность, в свою очередь, можно оценить, используя понятие среднего интеграла импульса напряжения. Средний интеграл импульса напряжения определялся путем усреднения интеграла импульса напряжения 27 осциллограмм для случая отсутствия дефектного состояния. Средний интеграл импульса напряжения на обмотке ВН был равен $8,75 \times 10^{-5}$ (Вб). Отклонение от среднего интеграла импульса напряжения от импульса к импульсу составляет величину, не превышающую $2 \%$. Можно считать, что это погрешность (шум) при определении интеграла импульса напряжения на обмотке ВН. Средний интеграл импульса напряжения на обмотке НН составил $7,52 \times 10^{-5}$ (Вб). Отклонение от среднего составило 1,6\%. Данные получены по 27 осциллограммам. Отклонение интеграла импульса напряжения от среднего для импульса-отклика по трем осциллограммам колебалось в пределах 3-4\%. Таким образом, можно полагать, что при зондировании погрешность (шум) лежит в пределах 3-4 \% от интеграла импульса. Поэтому для оценки состояния обмотки принимаем тот факт, что если для нормограммы и дефектограммы интеграл импульса напряжения отличаются более чем на $5 \%$ (более, чем шум), то это отклонение можно считать индикатором наличия дефектного состояния в обмотке трансформатора.

\section{Исследование чувствительности при наличии дефекта} типа «межвитковое короткое замыкание» в обмотке ВН

Для сравнения чувствительности предлагаемого комплекса и комплекса FRAX в обмотке BH фазы C был организован дефект типа «межвитковое короткое замыкание». Внешний вид обмотки с дефектом приведен на рис. $4,5$. 


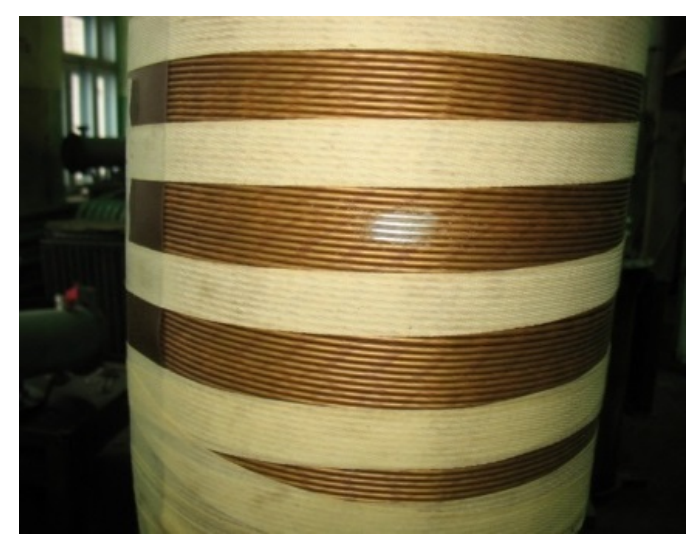

Рис. 4. Вид высоковольтной обмотки с дефектом типа «межвитковое короткое замькание»

Fig. 4. View of high voltage winding with fail of short circuit turn type

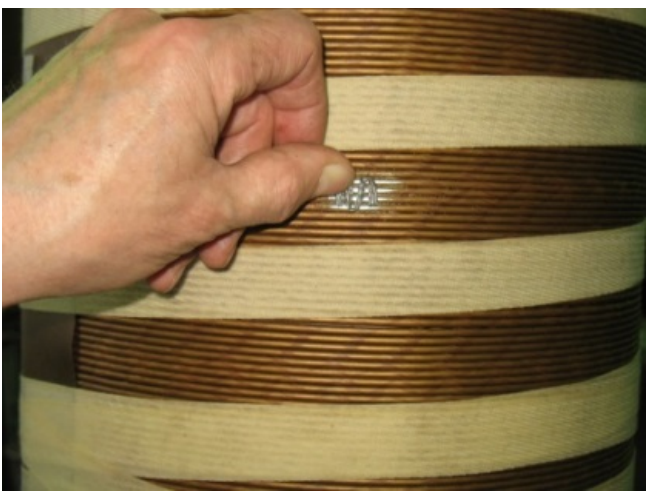

Pис. 5. Дефект типа «межвитковое короткое замыкание» на обмотке ВН

Fig. 5. Fail of short circuit turn type at the high voltage winding

Перед импульсным зондированием был проведен контроль состояния обмотки путем измерения сопротивления. Сопротивление до закоротки трех витков составило соответственно: «А-С» - 0,1046 Ом,
«В-C» - 0,1042 Ом, после закоротки «А-C» 0,1046 Ом, «В-С»-0,1043 Ом (измерено миллиомметром типа ПТФ-1). Таким образом, измерением сопротивления наличие трех короткозамкнутых витков не обнаруживается.

Диагностика наносекундным импульсом. На рис. 6 для примера приведены результаты сравнения сигналов отклика на наносекундный импульс при отсутствии дефектов - синяя кривая, и сигнал в случае межвиткового короткого замыкания - красная кривая. Для всех случаев нейтраль заземлена, остальные выводы обмоток находятся в режиме холостого хода.

Обработка осциллограмм по разработанной программе показывает, что интеграл разности импульсов напряжения нормограммы и дефектограммы равен $3,4 \times 10^{-6}$ (в диапазоне времени $0 \ldots 2,2 \times 10^{-6} \mathrm{c}$ ), интеграл импульса напряжения нормограммы (в диапазоне времени $\left.0 . .2,2 \times 10^{-6} \mathrm{c}\right)-1,01 \times 10^{-5}$, интеграл разности импульсов напряжения нормограммы и дефектограммы в процентах от интеграла импульса напряжения отклика трансформатора равен $34 \%$. Этот результат показывает, что использование предложенной методики обработки осциллограмм позволяет при закороченных трех витках обмотки ВН зарегистрировать значимое отличие между нормограммой и полученной дефектограммой. В табл. 2 приведены результаты обработки осциллограмм по приведенной выше методике для различных комбинаций приложения зондирующего импульса и отклика. Измерение сопротивления обмоток ВН омметром не выявило никакого различия между неповрежденной обмоткой и обмоткой с тремя закороченными витками при общем количестве витков обмотки ВН 1312, в то время как методом наносекундных импульсов по разработанной методике фиксируется различие в диапазоне от 35 до $55 \%$, которое зависит от комбинации обмотки, к которой прикладывается зондирующий импульс, и обмотки, на которой регистрируется импульс-отклик.

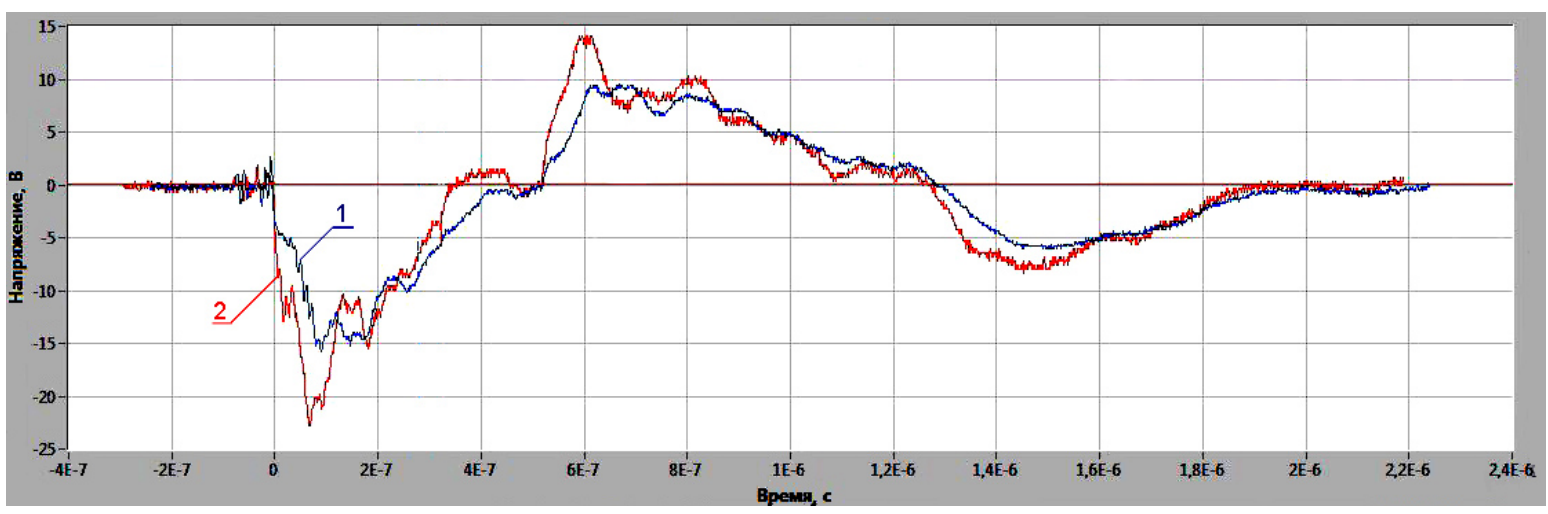

Pис.6. Зондирующий импульс подается на обмотку фазы $A$, сигнал отклика регистрируется на фазе а

Fig. 6. Probing impulse is applied to winding of phase A, response is measured at the phase a

Из табл. 2 видно, что наилучшая чувствительность имеет место при комбинации, когда зондирующий импульс, подаваемый на обмотку ВН, и импульсотклик, получаемый с обмотки НН, снимаются с пары обмоток одной фазы. Например, если дефект в об- мотке фазы $\mathrm{C}$, то наибольший интеграл разности напряжения импульсов-откликов получается, когда зондирующий импульс подаем на обмотку ВН фазы С, а отклик получаем с обмотки НН фазы с. 
Таблица 2. Отличие нормограмм от дефектограмм при различных комбинациях приложения зондирующего импульса и отклика

Table 2. Difference of normograms and failgrams at various combinations of supply points for probing impulse and response

\begin{tabular}{|c|c|c|c|}
\hline 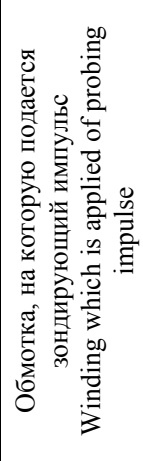 & 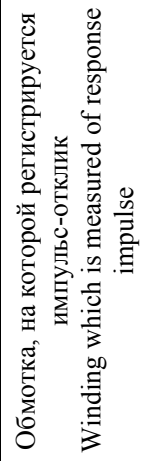 & 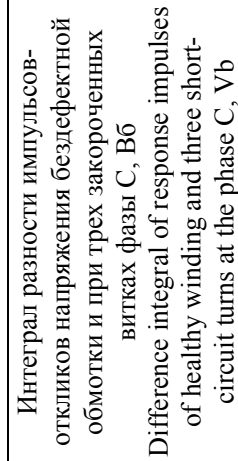 & 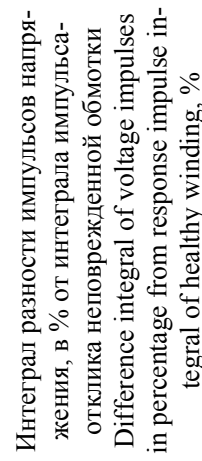 \\
\hline $\mathrm{A}$ & $\mathrm{b}$ & $8,7 \times 10^{-7}$ & 45 \\
\hline A & $\mathrm{c}$ & $8,9 \times 10^{-7}$ & 47 \\
\hline B & $\mathrm{c}$ & $9,3 \times 10^{-7}$ & 47 \\
\hline $\mathrm{C}$ & $\mathrm{a}$ & $8,7 \times 10^{-7}$ & 44 \\
\hline $\mathrm{C}$ & $\mathrm{c}$ & $3,5 \times 10^{-6}$ & 55 \\
\hline
\end{tabular}

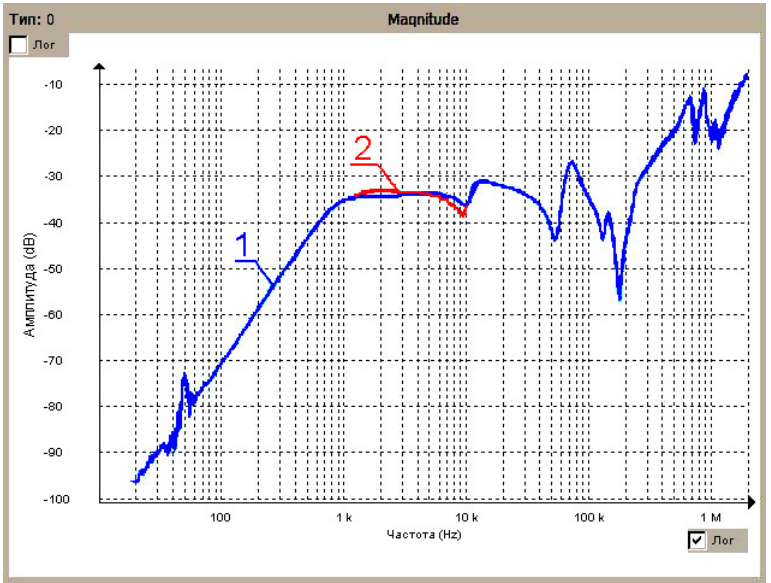

Рис. 7. Зондируюший импульс подается на обмотку фазы A, сигнал отклика регистрируется на фазе а

Fig. 7. Probing impulse is applied to the winding of phase A, response is measured at the phase a
Диагностика прибором FRAX-150. На рис. 7 приведены результаты измерений, проведенных методом FRA.

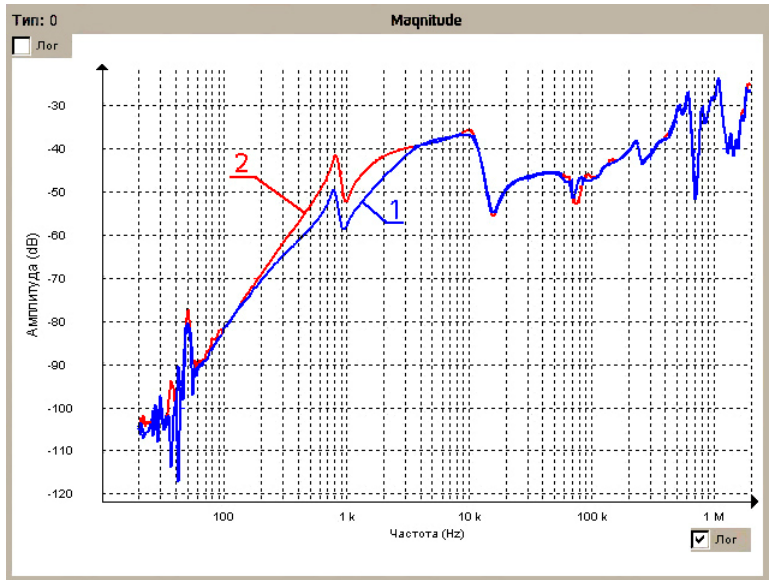

Pис. 8. Зондирующий импульс подается на обмотку фазы A, сигнал отклика регистрируется на фазе с

Fig. 8. Probing impulse is applied to the winding of phase A, response is measured at the phase $c$

Результаты, полученные с применением метода FRA, показывают, что наилучшая чувствительность реализуется при комбинации, когда зондирующее напряжение и отклик снимаются с пары обмоток одной фазы, как и в случае с методом наносекундных импульсов. Отличие в амплитудно-частотной характеристике наблюдаются в диапазоне частот от 200 Гц до 300 кГц. Метод FRA и метод наносекундных импульсов чувствительны к коротким замыканиям нескольких витков обмотки ВН и позволяют их обнаружить.

\section{Диагностика дефекта типа «аксиальное смещение витков» методом наносекундных импульсов}

Для исследования чувствительности методов при наличии дефекта типа «аксиальное смещение витков» на обмотке фазы А был организован указанный дефект, охватывающий 14 витков. Внешний вид обмотки ВН с дефектом типа «аксиальное смещение витков» показан на рис. 9.

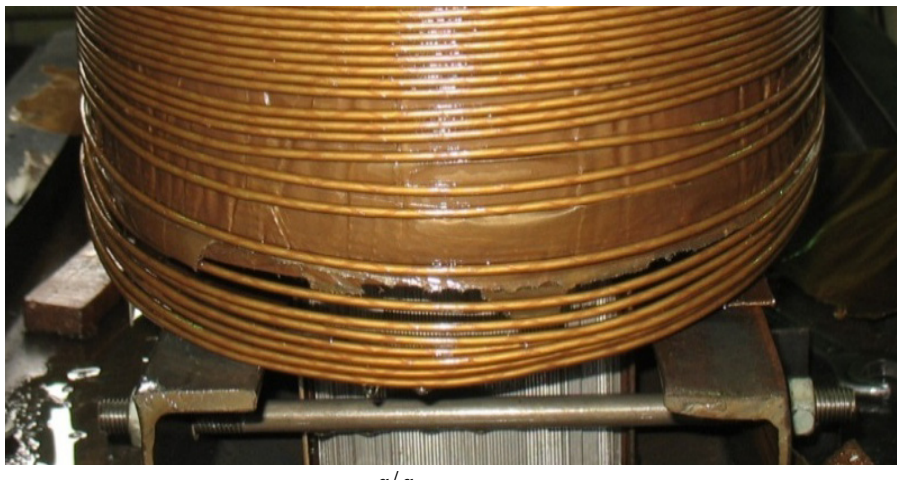

$a / a$

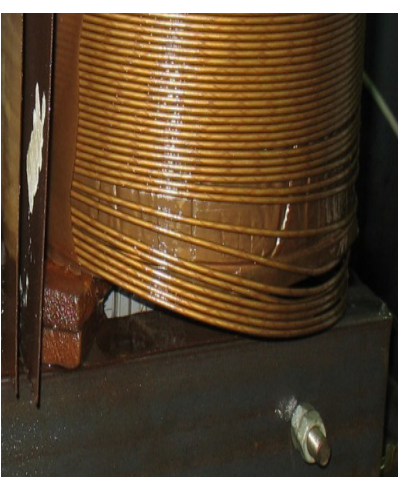

$\sigma / b$

Рис. 9. Внешний вид высоковольтной обмотки фазы А с дефектом типа «аксиальное смещение витков»: a) фронтальный вид катушки трансформатора, б) вид катушки сбоку

Fig. 9. View of high voltage phase A containing a fail type axial shift of turns: a) front view of winding, b) side view of the winding 
Ниже приведены результаты сравнения сигналов отклика на наносекундный импульс при отсутствии дефектов - синяя кривая, и сигнал в случае аксиального смещения витков - красная кривая, при различных комбинациях места подачи зондирующего им- пульса и места регистрации отклика. Для всех случаев нейтраль заземлена, остальные выводы обмоток находятся в режиме холостого хода. На рис. 10 приведены совмещенные осциллограммы-отклики, полученные с обмоток фазы а.

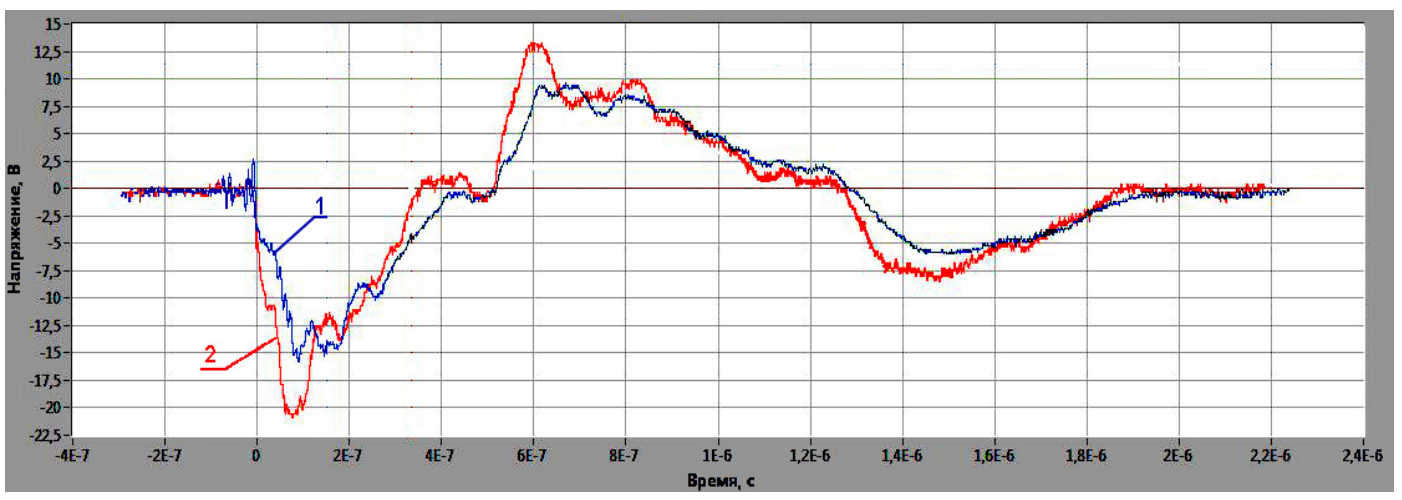

Рис. 10. Зондирующий импульс подается на обмотку фазы А, сигнал отклика регистрируется на фазе а

Fig. 10. Probing impulse is applied to the winding of phase A, response is measured at the phase a

В табл. 3 приведены результаты обработки осциллограмм аналогичные результатам, отраженным в табл. 2, для случая «аксиальное смещение витков» в обмотке ВН фазы А. Обработка сигналов проводилась по приведенной выше методике: разность интегралов имульсов-откликов для неповрежденной обмотки и обмотки со смещенными витками (рис. 10) сравнивался с интегралом импульса-отклика зондирующего сигнала.

Таблица 3. Отличие нормограмм от дефектограми при различных комбинациях приложения зонди рующего импульса и отклика при аксиальном смещении 14 витков высоковольтной обмотки фазы A

Table 3. Difference of normograms and failgrams at various combinations of applied points for probing impulse and response at the axial shift of 14 turns of high voltage phase $A$

\begin{tabular}{|c|c|c|c|}
\hline 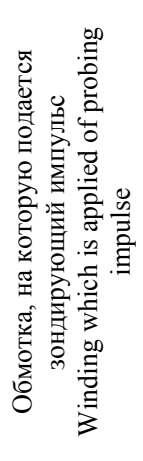 & 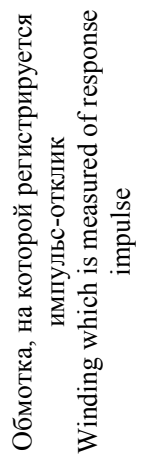 & 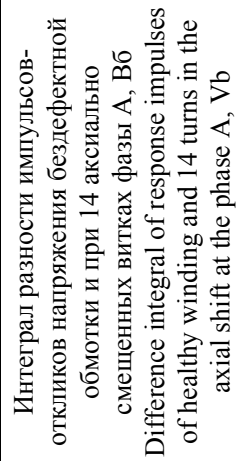 & 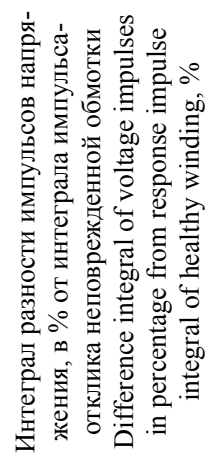 \\
\hline$\overline{\mathrm{A}}$ & a & $3,4 \times 10^{-6}$ & \\
\hline & c & $9,5 \times 10$ & \\
\hline & & $9,5 \times 10^{-7}$ & \\
\hline
\end{tabular}

Результаты табл. 3 показывают значимое отличие импульсов-откликов нормограмм от дефектограмм. Установить предел чувствительности в данном случае вызывает затруднения, т. к. ситуация со смещением витков приводит к изменению соотношений емкостей практически во всех фазах и существенно зависит от геометрических размеров катушек трансформатора и количества сдвинутых в аксиальном направлении витков. По-видимому, для каждого типа трансформатора это будет индивидуальный параметр.

\section{Диагностика дефекта типа «аксиальное смещение витков» методом FRA с применением прибора FRAX-150}

На рис. 11 приведены результаты измерений методом FRA для случая: зондирующий сигнал прикладывается к обмотке ВН А, сигнал-отклик снимается с обмотки НН а. Сигналы наложены для бездефектной обмотки и для обмотки со смещенными в аксиальном направлении 14 витками обмотки ВН фазы А.

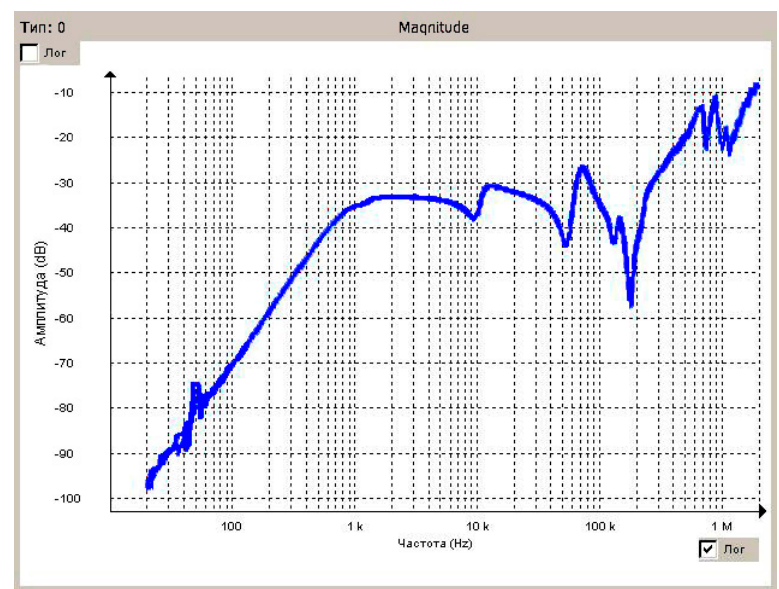

Рис. 11. Зондирующий импульс подается на высоковольтную обмотку фазы А, сигнал отклика регистрируется на фазе а

Fig. 11. Probing impulse is applied to the winding of phase $A$, response is measured at the phase a

На рис. 12 приведены результаты измерений методом FRA для случая: зондирующий сигнал прикладывается к обмотке ВН А, сигнал-отклик снимается с 
обмотки НН с. Сигналы наложены для бездефектной обмотки и для обмотки со смещенными в аксиальном направлении 14 витками обмотки ВН фазы А.

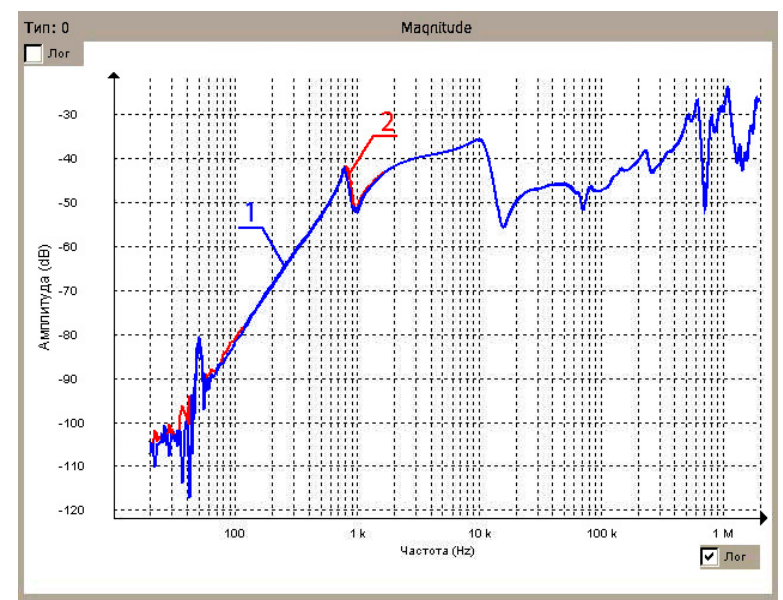

Pис. 12. Зондирующий импульс подается на обмотку фазы А, сигнал отклика регистрируется на фазе с

Fig. 12. Probing impulse is applied to the winding of phase $A$, response is measured at the phase $c$

На рис. 13-15 приведены аналогичные результаты измерений методом FRA для других случаев приложения зондирующего сигнала и сигнала-отклика. Во всех случаях сигналы наложены для бездефектной обмотки и для обмотки со смещенными в аксиальном направлении 14 витками обмотки ВН фазы А.

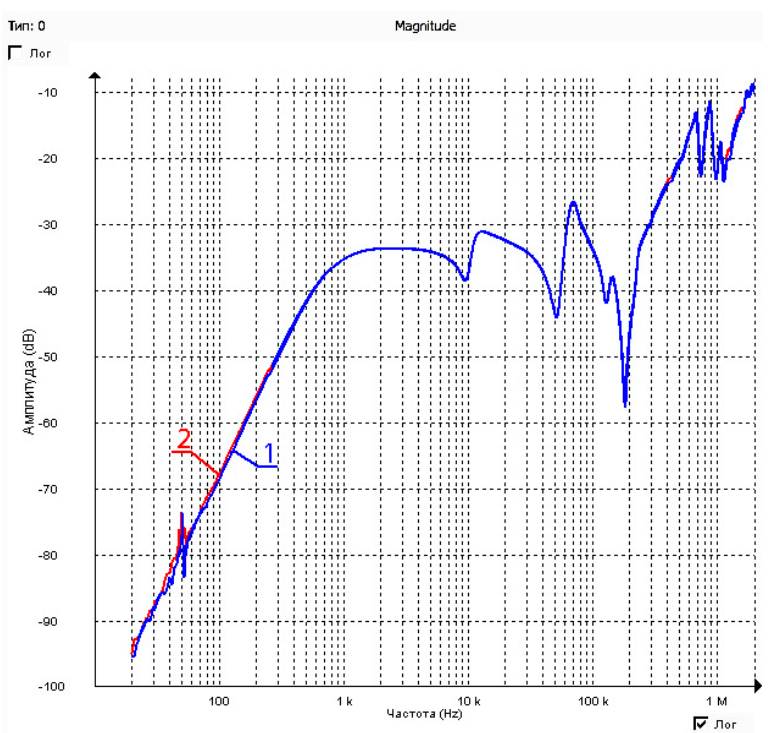

Pис. 13. Зондируюший импульс подается на обмотку фазы $B$, сигнал отклика регистрируется на фазе $b$

Fig. 13. Probing impulse is applied to the winding of phase $B$, response is measured at the phase $b$

\section{Обсуждение результатов}

Из приведенных результатов контроля состояния обмоток по методу FRA следует, что выявить характерное различие между ними довольно трудно. Аналогичные картины наблюдаются при других сочетаниях схем. Делать вывод о наличии дефектного со- стояния обмотки на основании приведенных выше результатов анализа АЧХ, полученных при измерении комплексом FRA, крайне затруднительно. Вероятность ошибочного диагноза, поставленного по результатам анализа АЧХ, достаточно высока.

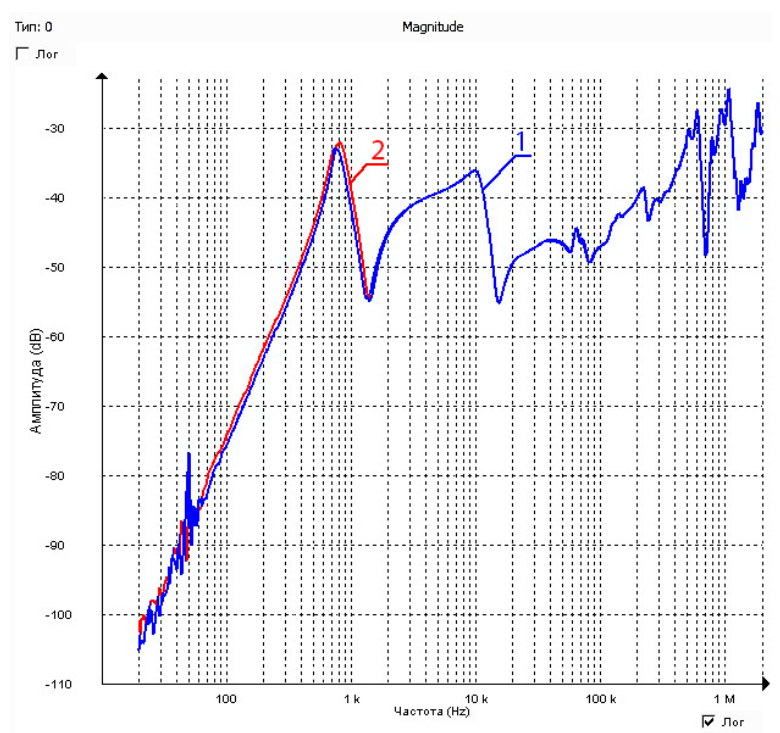

Рис. 14. Зондирующий импульс подается на обмотку фазы B, сигнал отклика регистрируется на фазе $c$

Fig. 14. Probing impulse is applied to the winding of phase $B$, response is measured at the phase $c$

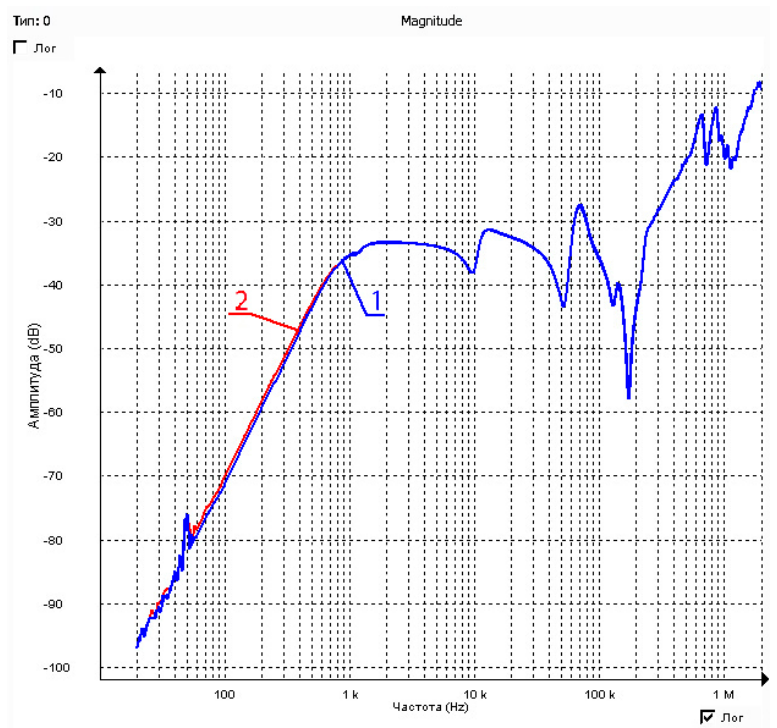

Pис. 15. Зондирующий импульс подается на обмотку фазы C, сигнал отклика регистрируется на фазе с

Fig. 15. Probing impulse is applied to the winding of phase $C$, response is measured at the phase $c$

На основании результатов исследований можно сделать вывод о том, что метод наносекундных импульсов и метод FRA примерно с одинаковой чувствительностью реагируют на межвитковые замыкания, но первый более чувствителен в отношении смещения витков в высоковольтной обмотке трансформатора. Так, метод наносекундных импульсов позволяет обнаруживать смещение 14 витков высоко- 
вольтной обмотки, тогда как методом FRA такой дефект выявить не удалось.

Таким образом, есть все основания заключить, что эффективность контроля состояния обмоток оказывается как минимум не хуже, а в ряде случае выше технологии FRA. Объяснение состоит в том, что зондирующий импульс наносекундной длительности с коротким фронтом прямоугольной формы насыщен высокочастотной составляющей в диапазоне сотни кГц вплоть до десятков МГц. Более высокая граница частотного диапазона обеспечивает более высокую интенсивность переходного процесса по сравнению с методом FRA, предел частотного диапазона которого ограничивается значением 1-2 МГц.

\section{Выводы по результатам исследования}

Разработан диагностический комплекс для контроля состояния обмоток трансформаторов, реализованный на основе генератора зондирующих импульсов по схеме Ю.В. Введенского, который позволяет формировать импульс длительностью в диапазоне 60-500 нс с фронтом 25 нс на нагрузке с произвольным импедансом.

Показано, что при импульсном методе дефектографирования состояния обмоток трансформатора для обнаружения смещения витков обмотки и короткого замыкания витков высокая чувствительность достигается при длительности импульса 60-500 нс с фронтом 15-20 нс.

\section{СПИСОК ЛИТЕРАТУРЫ}

1. Соколов В.В. Ранжирование состаренного парка силовых трансформаторов по техническому состоянию // Современное состояние и проблемы диагностики силового электрооборудования: Материалы IV Всероссийской научно-технической конференции. - Новосибирск, 26-28 сентября, 2006. - С. 7-18.

2. Pettersson L., Fantana N.L., Sunderman U. Assessment ranking of power transformers using condition based evaluation, a new approach // CIGRE Paris Conference. - Paris, France, 1998. Paper 12-204.

3. Осотов В.Н. О методологии оценки состояния электрооборудования с большим сроком службы // Современное состояние и проблемы диагностики силового электрооборудования: Материалы IV Всероссийской научно-технической конференции. - Новосибирск, 26-28 сентября, 2006. - С. 25-29.

4. Вдовико В.П. Диагностика высоковольтного электрооборудования и эффективность ее применения // Современное состояние и проблемы диагностики силового электрооборудования: Материалы IV Всероссийской научно-технической конференции. - Новосибирск, 26-28 сентября, 2006. - С. 34-41.

5. Лех В., Тымински Л. Новый метод индикации повреждений при испытании трансформаторов на динамическую прочность // Электричество. - 1966. - Т. 1. - № 1. - С. 77-81.

6. Аветиков Г.В., Левицкая Е.И., Попов Е.А. Импульсное дефектографирование трансформаторов на при испытаниях на электродинамическую стойкость // Электротехника - 1978. - № 4. - С. 53-57.

7. Конов Ю.С., Короленко В.В., Федорова В.П. Обнаружение повреждений трансформаторов при коротких замыканиях // Электрические станции - 1980. - № 7. - С. 46-48.

8. Количественная оценка результатов импульсного дефектографирования обмоток силовых трансформаторов / С.В. Аликин, А.А. Дробышевский, Е.И. Левицкая, М.А. Филатова // Электротехника. - 1990. - № 5. - С. 75-76.

9. Диагностика обмоток силовых трансформаторов методом низковольтных импульсов / С.В. Аликин, А.А. Дробышевский, Е.И. Левицкая, М.А. Филатова // Электротехника - 1991. № 12 . - C. $30-35$.
Экспериментально доказано, что технология импульсного дефектографирования механического состояния обмоток трансформаторов зондирующими импульсами наносекундной длительности обладает более высокой чувствительностью к обнаружению смещений обмоток трансформаторов в радиальном и аксиальном направлениях по сравнению с методом частотного анализа.

\section{Заключение}

Развитие дефектов обмоток происходит неконтролируемо и в результате заканчивается выходом трансформатора из строя. Возможность контролировать дефекты на ранней стадии развития как механического, так и электрического типа, обеспечивая своевременный вывод трансформаторного оборудования в ремонт, является одной из основных задач стоящих перед энергосистемами РФ. Не смотря на множество положительных сторон и рекомендаций СИГРЭ, результаты экспериментов, представленных в статье, подтверждают факт, что комплекс FRAX далеко не всегда оказывается в должной степени эффективным. Реальной альтернативой технологии FRA может оказаться диагностический комплекс, в основе работы которого лежит контроль состояния обмоток зондирующими импульсами наносекундной длительности. Эксперименты, выполненные на реальном трансформаторе, подтвердили перспективность этого подхода.

10. Хренников А.Ю., Киков О.М. Диагностика силовых трансформаторов в Самараэнерго методом низковольтных импульсов // Электрические станции. - 2003. - № 11. - С. 49-51.

11. Хренников А.Ю. Опыт обнаружения остаточных деформаций обмоток силовых трансформаторов // Энергетик. - 2003. № 7. - C. 18-20.

12. Diagnosis of interturn faults of single-distribution transformers under controlled conditions during energization / D. Guillen, J. Olivares-Galvan, R. Escarela-Perez, D. Granados-Lieberman, E. Barocio // Measurement. - 2019. - V. 141. - P. 24-36.

13. High frequency electric circuit modeling for transformer frequency response analysis studies / X. Zhaoa, C. Yaoa, A. Abu-Siadab, R. Liaoa // Electrical Power and Energy Systems. - 2019. V. 111. - P. 351-368.

14. Diagnostic of transformer winding deformation fault types using continuous wavelet transform of pulse response / Q. Cheng, Z. Zhao, C. Tang, G. Qian, S. Islam // Measurement. - 2019. V. 140. - P. 197-206.

15. Velasquez R., Lara J., Melgar A. Converting data into knowledge for preventing failures in power transformers // Engineering Failure Analysis. - 2019. - V. 101. - P. 215-229.

16. Zarkovi M., Stojkovi Z. Analysis of artificial intelligence expert systems for powertransformer condition monitoring and diagnostics // Electric Power Systems Research. - 2017. V. 149. - P. 125-136.

17. Senobari R., Sadeh J., Borsi H. Frequency response analysis (FRA) of transformers as a tool for fault detection and location: a review // Electric Power Systems Research. - 2019. - V. 155. - P. 172-183.

18. Investigating the applicability of the finite integration technique for studying the frequency response of the transformer winding / P. Hillenbrand, S. Tenbohlen, M. Samimi, A. Shayegani, A. Mohseni, J. Faiz // Electrical Power and Energy Systems. - 2019. - V. 110. - P. 411-418.

19. Брошюра СИГРЭ № 342. Mechanical condition assessment of transformer windings using frequency response analysis (FRA), WG A2.26, 2008.

20. Ларин В.С. Анализ частотных характеристик для локализации коротких замыканий в обмотках трансформаторов // Электричество - 2018. - № 4. - С. 14-25. 
21. Lavrinovich V.A., Lavrinovich A.V., Mytnikov A.V. Development of advanced control state technology of transformer and electric motor windings based on pulsed method // International Journal on Technical and Physical Problems of Engineering. - 2012. - V. 4. - № 4. - P. 149-153.

22. Lavrinovich V.A, Isaev Y.N., Mytnikov A.V. Advanced control state technology of transformer windings // International Journal on Technical and Physical Problems of Engineering. - 2013. V. 5. - № 4. - P. 94-98.
23. Lavrinovich V.A, Isaev Y.N., Mytnikov A.V. Modeling of state control procedure of power transformer winding by short probe pulses // International Journal on Technical and Physical Problems of Engineering. - 2014. - V. 6. - № 1. - P. 145-147.

24. Lavrinovich V.A., Mytnikov A.V. Development of pulsed method for diagnostics of transformer windings based on short probe impulse // IEEE Translation on Dielectric Electrical Insulation 2015. - V. 22. - № 4. - P. 2041-2045.

Поступила 15.10.2020 2.

\section{Информация об авторах}

Лавринович А.В., инженер 1 категории, отдел по высоковольтному оборудованию, Томский научноисследовательский и проектный институт нефти и газа.

Mытников A.B., кандидат технических наук, доцент Инженерной школы энергетики Национального исследовательского Томского политехнического университета. 
UDC 621.314.21.027.3.045:681.518.54

\title{
DIAGNOSTICS FACILITY FOR EFFECTIVE CONTROL OF WINDING CONDITION OF HIGH VOLTAGE TRANSFORMERS
}

\author{
Alexey V. Lavrinovich1, \\ lavrinovich86@yandex.ru
}

\author{
Alexey V. Mytnikov², \\ mytnikov66@mail.ru \\ 1 Tomsk Research and Design Institute of Oil and Gas, \\ 72, Mira avenue, Tomsk, 634027, Russia. \\ 2 National Research Tomsk Polytechnic University, \\ 30, Lenin avenue, Tomsk, 634050, Russia.
}

The importance of the research is driven by the fact that all stages of production and processing of geological resources include a large-scale technological complex, in which the dominant role is played by electric power systems that ensure the operation of all the main components and assemblies. A significant class of high-voltage equipment of such systems is distribution power transformers and shunt reactors. Failure of transformer equipment causes a rapid shutdown of the technological cycle, an emergency and financial losses. This can be avoided by ensuring the reliable operation of electrical equipment by conducting quality diagnostics to assess the residual life of a transformer or reactor. Standard technologies, such as measurements of direct current resistance, absorption coefficient and dielectric loss tangent do not allow detecting a winding defect at an early stage of development, and therefore cannot be considered effective. In almost all world energy systems, the FRA technological complex is considered to be promising and recommended for use, which is a technical implementation of the method of analyzing frequency characteristics. However, this technology encounters difficulties of a technical and organizational nature when implemented in Russian energy systems. The instrument base and software do not always meet the requirements and standards for testing electrical equipment of the grids of the Russian Federation. As a result, there are a high percentage of bad conclusions about the results of monitoring. The issue is complicated due to the fact that the patent for the use of FRA technology belongs to the institutes of the USA, Canada and several European countries. Taking into account the unfriendly political situation, a number of sanctions and restrictions on the export of machinery and technologies, further use of FRA technology may be problematic. Modern technology corresponding to the requirements does not exist in the Russian Federation. In this regard, the development of a reliable diagnostic complex that takes into account the technical, climatic and other features of the electric power systems of the Russian Federation and allows effective monitoring of the state of the windings taking into account the specifics of domestic transformer equipment is an urgent task.

The main goal of the work is to research the effectiveness of the diagnostic complex based on probing pulses of nanosecond duration on real transformer equipment. To conduct a comparative analysis of the results, monitoring the status of the transformer windings of the developed complex and the FRA technological complex.

Object of the research is real transformer TM-160/10, fails such as short-circuited turns and mechanical displacement of the windings were created.

Methods: experimental measurements of probing pulses and response in both technologies - pulsed and FRA. Comparison of condition control efficiency for both methods. Using Tektronix electronic oscilloscopes of the TDS-1012 type, a probe and a response pulse were recorded. The measurements were carried out for the same defective states of the winding using the FRA complex.

Results. On a real transformer, the efficiency of the developed diagnostic complex for monitoring the status of transformer windings based on nanosecond probe pulses was experimentally studied. It is shown that the efficiency of monitoring the status of transformer windings with the help of the developed complex is no worse, and in some cases exceeds the efficiency of the FRA complex.

\section{Key words:}

Winding condition control, pulsed method, probing impulse, winding fail, control efficiency.

\section{REFERENCES}

1. Sokolov V.V. Ranzhirovanie sostarennogo parka silovykh transformatorov po tekhnocheskomu sostoyaniyu [Ranking of the aged power transformer park due to technical technical condition]. Sovremennoe sostovanie i problem diagnostiki silovogo elektrooborudovaniya. Materialy IV Vserossiyskoy nauchno-tekhnicheskoy konferentsii [Modern condition and problems of power electrical equipment diagnostics. Proc. of IV All-Russian scientific conference]. Novosibirsk, Russia, 2006. pp. 7-18.

2. Pettersson L., Fantana N.L., Sunderman U. Assessment ranking of power transformers using condition based evaluation, a new approach. CIGRE Paris Conference. Paris, France, 1998. Paper 12-204.

3. Osotov V.N. O methodologii oschenki sostoyaniya elektrooborudovaniya $\mathrm{s}$ bolshim srokom sluzhby [About methodology of electrical equipment estimation with large term in service]. Sovremennoe sostoyanie i problem diagnostiki silovogo elektrooborudovaniya. Materialy IV Vserossiyskoy nauchno-tekhnicheskoy konferentsii [Modern condition and problems of power electrical equipment diagnostics. Proc. of IV All-Russian scientific conference]. Novosibirsk, Russia, 2006. pp. 25-29.

4. Vdoviko V.P. Diagnostika vysokovoltnogo elektrooborudovaniya I effektivnost ee primeneniya [Diagnostics of high voltage equipment and effectiveness of its application]. Sovremennoe sostoyanie i problem diagnostiki silovogo elektrooborudovaniya. Materialy IV Vserossiyskoy nauchno-tekhnicheskoy konferentsii [Modern condition and problems of power electrical equipment diagnostics. Proc. of IV All-Russian scientific conference]. Novosibirsk, Russia, 2006. pp. 34-41.

5. Lekh V., Tyminskiy L. New method of fail diagnostics at the dynamic reliability transformer test. Electricity, 1966, vol. 1, no. 1, pp. 77-81. In Rus.

6. Avetikov G.V., Levitskaya E.I., Popov E.A. Impulsed defectography of transformers at the electrodynamic reliability test. Electrotechnic, 1978, no. 4, pp. 53-57. In Rus.

7. Konov Yu.S., Korolenko V.V., Fedorova V.P. Fail detection of transformers due to short circuit. Electric Power Stations, 1980, no. 7, pp. 46-48. In Rus. 
8. Alikin S.V., Drobyscevskiy A.A., Levitschaya E.I., Filatova M.A. Quantitative assessment of the of pulse defectography results of power transformer windings. Electrotechnic, 1990, no. 5, pp. 75-76. In Rus.

9. Alikin S.V., Drobyscevskiy A.A., Levitschaya E.I., Filatova M.A. Power transformer winding diagnostics by low-voltage pulsed method. Electrotechnic, 1991, no. 12, pp. 30-35. In Rus.

10. Khrennikov A.Yu., Kikov O.M. Power transformer diagnostics by low-voltage pulsed method in Samaropenergo. Electric Power Stations, 2003, no. 11, pp. 49-51. In Rus.

11. Khrennikov A.Yu. Experience of mechanical deformation detection of power transformer windings. Energetic, 2003, no. 7, pp. 18-20. In Rus.

12. Guillen D., Olivares-Galvan J., Escarela-Perez R., GranadosLieberman D., Barocio E. Diagnosis of interturn faults of singledistribution transformers under controlled conditions during energization. Measurement, 2019, vol. 141, pp. 24-36.

13. Zhaoa X., Yaoa C., Abu-Siadab A., Liaoa R. High frequency electric circuit modeling for transformer frequency response analysis studies. Electrical Power and Energy Systems, 2019, vol. 111, pp. 351-368.

14. Cheng Q., Zhao Z., Tang C., Qian G., Islam S. Diagnostic of transformer winding deformation fault types using continuous wavelet transform of pulse response. Measurement, 2019, vol. 140, pp. 197-206.

15. Velasquez R., Lara J., Melgar A. Converting data into knowledge for preventing failures in power transformers. Engineering Failure Analysis, 2019, vol. 101, pp. 215-229.

16. Zarkovi M., Stojkovi Z. Analysis of artificial intelligence expert systems for powertransformer condition monitoring and diagnostics. Electric Power Systems Research, 2017, vol. 149, pp. $125-136$
17. Senobari R., Sadeh J., Borsi H. Frequency response analysis (FRA) of transformers as a tool for fault detection and location: a review. Electric Power Systems Research, 2019, vol. 155, pp. 172-183.

18. Hillenbrand P., Tenbohlen S., Samimi M., Shayegani A., Mohseni A., Faiz J. Investigating the applicability of the finite integration technique for studying the frequency response of the transformer winding. Electrical Power and Energy Systems, 2019, vol. 110, pp. 411-418.

19. Brochure of SIGRE № 342. Mechanical condition assessment of transformer windings using frequency response analysis (FRA), WG A2.26, 2008

20. Larin V.S. Frequent characteristic analysis for short circuit location in transformer windings. Electricity, 2018, no. 4, pp. 14-25. In Rus.

21. Lavrinovich V.A., Lavrinovich A.V., Mytnikov A.V. Development of advanced control state technology of transformer and electric motor windings based on pulsed method. International Journal on Technical and Physical Problems of Engineering, 2012, vol. 4, no. 4, pp. 149-153.

22. Lavrinovich V.A., Isaev Y.N., Mytnikov A.V. Advanced control state technology of transformer windings. International Journal on Technical and Physical Problems of Engineering, 2013, vol. 5, no. 4, pp. 94-98.

23. Lavrinovich V.A., Isaev Y.N., Mytnikov A.V. Modeling of state control procedure of power transformer winding by short probe pulses. International Journal on Technical and Physical Problems of Engineering, 2014, vol. 6, no. 1, pp. 145-147.

24. Lavrinovich V.A., Mytnikov A.V. Development of pulsed method for diagnostics of transformer windings based on short probe impulse. IEEE Translation on Dielectric Electrical Insulation, 2015, vol. 22, no. 4, pp. 2041-2045.

Received: 15 October 2020.

\section{Information about the authors}

Alexey V. Lavrinovich, engineer of first category, Tomsk Research and Design Institute of Oil and Gas. Alexey V. Mytnikov, Cand. Sc., associate professor, National Research Tomsk Polytechnic University. 